ARTICLE OPEN

\title{
Attempts to replicate genetic associations with schizophrenia in a cohort from north India
}

\author{
Suman Prasad ${ }^{1}$, Triptish Bhatia ${ }^{2}$, Prachi Kukshal ${ }^{1}$, Vishwajit L. Nimgaonkar ${ }^{3}$, Smita N. Deshpande ${ }^{2}$ and B. K. Thelma ${ }^{1}$
}

Schizophrenia is a chronic, severe, heritable disorder. Genome-wide association studies, conducted predominantly among Caucasians, have indicated $>100$ risk alleles, with most significant SNPs on chromosome 6 . There is growing interest as to whether these risk alleles are relevant in other ethnic groups as well. Neither an Indian genome-wide association studies nor a systematic replication of GWAS findings from other populations are reported. Thus, we analyzed 32 SNPs, including those associated in the Caucasian ancestry GWAS and other candidate gene studies, in a north Indian schizophrenia cohort ( $n=1009$ patients; $n=1029$ controls) using a Sequenom mass array. Cognitive functioning was also assessed using the Hindi version of the Penn Computerized Neuropsychological Battery in a subset of the sample. MICB (rs6916394) a previously noted Caucasian candidate, was associated with schizophrenia at the $p=0.02$ level. One SNP, rs2064430, AHI1 (6q23.3, SZ Gene database SNP) was associated at the $p=0.04$ level. Other candidates had even less significance with rs6932590, intergenic ( $p=0.07)$; rs3130615, MICB ( $p=0.08)$; rs6916921, NFKBIL1 ( $p=0.08)$ and rs9273012, HLA-DQA1 $(p=0.06)$ and haplotypic associations $(p=0.01-0.05)$ of $6 p$ SNPs were detected. Of note, nominally significant associations with cognitive variables were identified, after covarying for age and diagnostic status. SNPs with $p<0.01$ were: rs3130375, with working memory $(p=0.007)$; rs377763, with sensorimotor $(p=0.004)$; rs6916921, NFKBIL 1 with emotion $(p=0.01)$. This relative lack of significant positive associations is likely influenced by the sample size and/or differences in the genetic architecture of schizophrenia across populations, encouraging population specific studies to identify shared and unique genetic risk factors for schizophrenia.

npj Schizophrenia (2017)3:28; doi:10.1038/s41537-017-0030-8

\section{INTRODUCTION}

Evidence for a genetic etiology of schizophrenia (SZ) is available from family, twin and adoption studies, and the heritability has been estimated at $\sim 70 \%{ }^{1{ }^{1} 2}$ However, it needs to be emphasized that these conclusions are based on data from Caucasian populations and little is known about whether they are similar in other racial groups. Overall, family based linkage analyses in all populations provided scant evidence for replicable risk factors. ${ }^{3}$ Though some genetic association studies relying on candidate neurotransmitters indicated suggestive associations, the associations were difficult to replicate. ${ }^{4}$ Encouragingly, Genome-wide association studies (GWASs) utilizing very large sets of samples and common variants densely covering the entire genome have yielded more consistent results. ${ }^{5-7}$ The most significantly associated SNPs were noted on chromosome 6p21-24 in an initial GWAS, while other groups reported associations with copy number variants and with cognitive endophenotypes. ${ }^{8-12}$ These too were largely performed in only Caucasian populations. More recently, Phase I and II of Schizophrenia Working Group of the psychiatric genomics consortium (PGC) reported significant associations at 108 loci for SZ in a sample of 21,000 cases and 38,000 controls $^{13}$ of European descent; the strongest associations were also noted in the major histocompatibility (MHC) region on chromosome 6 , known to be involved in immune function, ${ }^{14}$ consistent with earlier association studies. ${ }^{15-17}$ More refined analyses indicated associations with a copy number variation incorporating the C4A locus which was supported by postmortem gene expression studies. ${ }^{18}$ Other associations, including genes related to Calcium channel functions and to histone methylation processes have been reported. ${ }^{5,19}$ The chromosome 6p21-24 region is gene dense and harbors the HLA (human leukocyte antigen) or MHC (major histocompatibility) locus which includes more than 100 immune response genes and several other genes unrelated to immune function. On the other hand, the chromosome $6 \mathrm{q}$ region harbors the AHI1 (human Abelson helper integration site-1) gene which has been shown to be associated with neurological, hematologic, and metabolic diseases. ${ }^{20,21}$ Variation in MHC genes could be associated with SZ, in part through impaired immune responses, increase related to predisposition to viral infection(s) ${ }^{22,}{ }^{23}$ neuroinflammation or even altered synaptic plasticity. ${ }^{24}$ Cognitive impairment, one of the deficits, central to SZ etiology ${ }^{25}$ has been observed consistently. A relatively high range of heritability (32-67\%) for General Cognitive Ability was observed in a meta-analysis of $>800,000$ subjects, likely due to differences in genetic and environmental effects, confirming that many cognitive phenotypes are under strong genetic influences. ${ }^{26}$ It is thought by many investigators that aspects of cognitive impairment may be endophenotypes or intermediate phenotypes that are more directly related to the genetic risk factors than the clinical illness of SZ itself. Almost all of these studies have been performed with samples of Caucasian ancestry.

\footnotetext{
${ }^{1}$ Department of Genetics, University of Delhi South Campus, New Delhi 110 021, India; ${ }^{2}$ Department of Psychiatry, Center of Excellence in Mental Health, PGIMER- Dr Ram

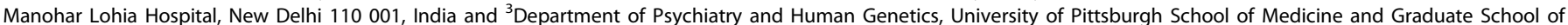
Public Health, Pittsburgh, PA 15213, USA

Correspondence: Smita N. Deshpande, (smitadeshp@gmail.com) or B. K. Thelma (thelmabk@gmail.com)
}

Received: 7 April 2017 Revised: 18 July 2017 Accepted: 24 July 2017

Published online: 30 August 2017 
Studies in other populations have been initiated, but have lagged in sample size compared with the Caucasian ancestry samples. ${ }^{27}$ Though the diverse Indian populations are genetically more closer to Caucasian populations ${ }^{28-30}$ they are notably different based on admixture analysis and poor portability of tag SNPs. ${ }^{31,} 32 \mathrm{SZ}$ is prevalent in India, ${ }^{33}$ but relatively few gene mapping studies have been conducted. The population is neither included in the large GWASs and PGC- GWAS, nor are there any studies using polygenic risk scores based on PGC data. In past two decades, a number of hypothesis-driven candidate gene variations, focused mainly on genes from the neurotransmitter and signaling pathways, were tested for their association with SZ, as well as tardive dyskinesia, cognition and response to antipsychotic drugs in the Indian population (Table 1). The sample size in these studies varied (see reviews $\left.{ }^{34},{ }^{35}\right)$. Of these, the most significant associations were observed for rs715505 ( $p=0.00007)$ in synaptogyrin $-1^{36}$; and those with SNPs in NRG1 were replicated ${ }^{37,38}$ (Table 1). More recently, efforts to check for SNP frequencies across Asian populations have been made for SZ. ${ }^{39}$ A sub-group of these samples $(n=489)$ belong to South Asian (SAS) individuals comprising of Gujarati Indians, Pakistani Punjabis, Bangladeshi Bengalis, Sri Lankan Tamils, and Indian Telugus. Though the mean allele frequencies of the genetic variants did not significantly differ between these populations, the combination of these variants was suggested to increase the risk of $\mathrm{SZ}^{39}$ Of note, rs13194504 near C4A locus from the strongly associated $\mathrm{MHC}$ region ${ }^{18}$ was found to be monomorphic in all the SAS populations (including north Indian population, our unpublished observation) and therefore was not analyzed further in our sample set. On the other hand, there has been only one study from India which tested association of PGC SNPs with SZ. This study using a custom panel of Illumina 1536 SNPs including PGC SNPs tested 351 patients and 385 controls from north India; 436 patients, 401 controls

Table 1. Previously reported candidate gene studies showing significant association with SZ cohorts from Indian population

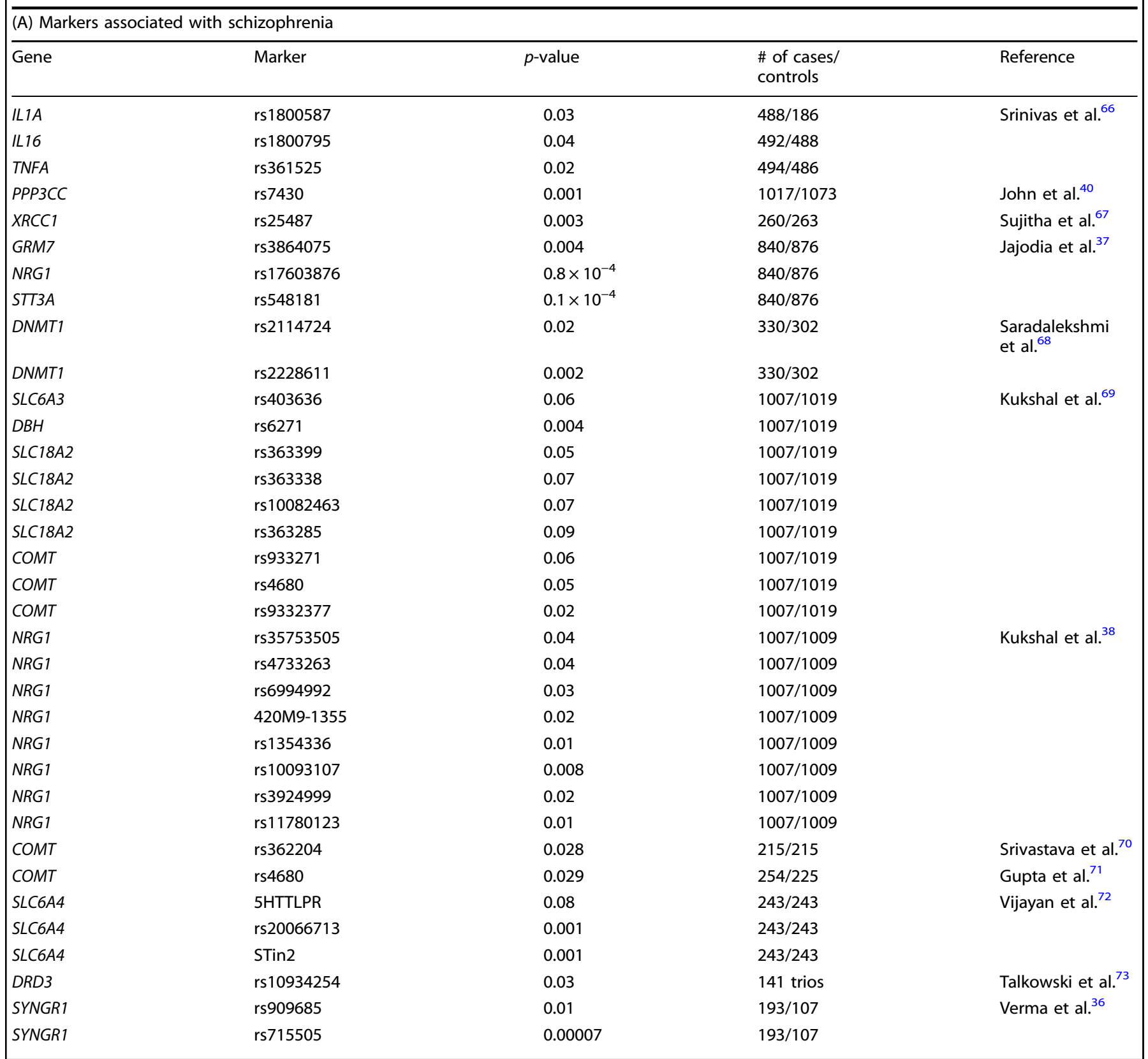




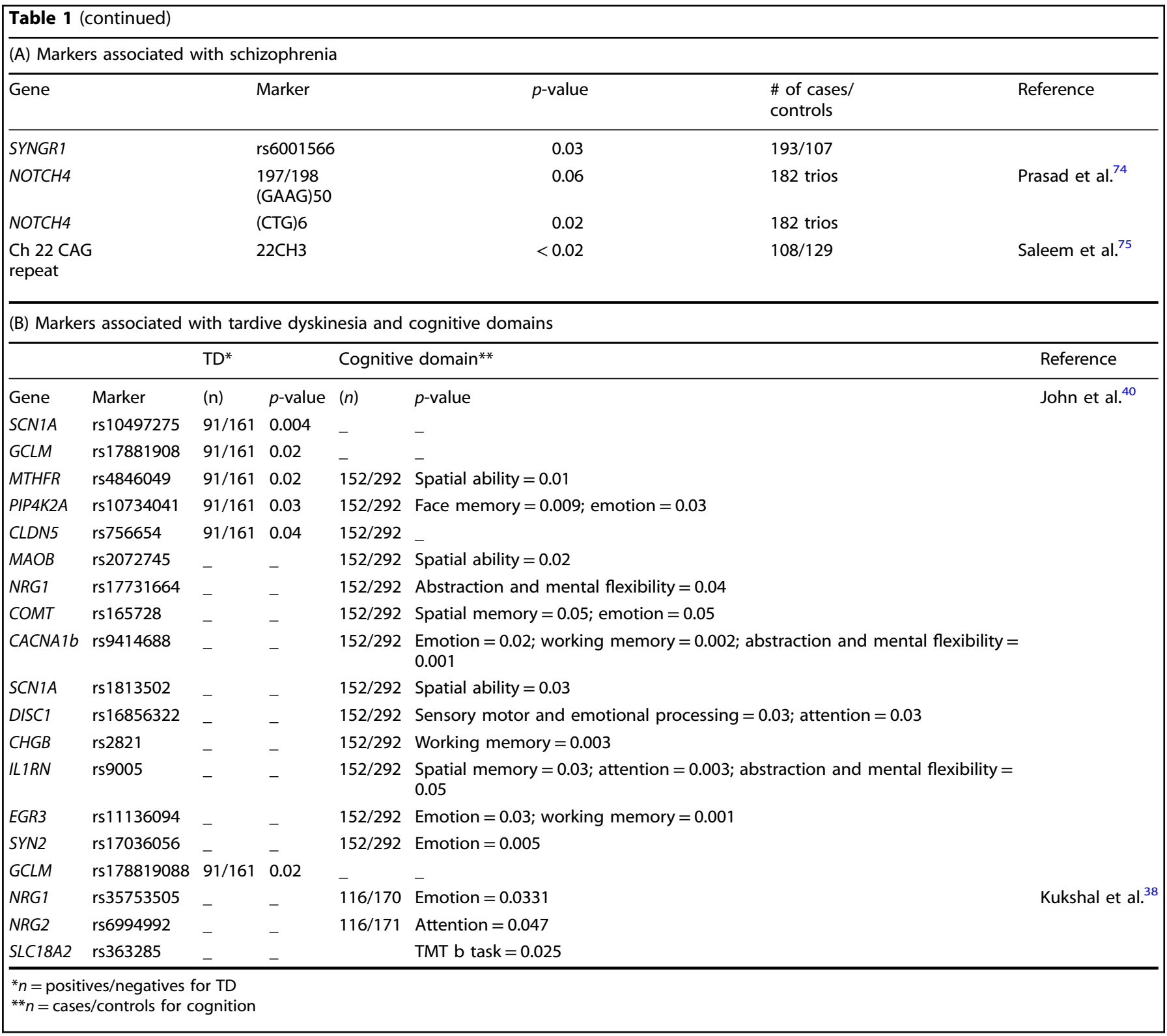

and 143 familial samples including complete and incomplete trio families from south India and identified a single PGC SNP rs548181 STT3A, (11q24.2) to be associated ( $p=1.39 \times 10^{-7}$, uncorrected; OR $(95 \% \mathrm{Cl})=0.308(0.181-0.524) .{ }^{37}$ In a different study of 35 miRSNPs with predicted functional relevance in $3^{\prime}$ UTRs of genes shown previously to be associated with SZ, a significant association of miRSNP rs7430 in PPP3CC with SZ in a north Indian cohort has been reported. ${ }^{40}$ Further, an association $(p=0.001)$ of miRSNP rs9414688 (CACNA1b) and rs11136094 (EGR3) with cognitive domains of abstraction and mental flexibility and working memory respectively was also reported therein. ${ }^{40}$ Association of rs10497275 $(p=0.004)$ in SCN1A, in a subset of TD cases was also observed in the same study. ${ }^{40}$ Finally, using a whole exome sequencing strategy in multiplex SZ families from India, rare variants in TAAR1 have been reported for the first time. ${ }^{41}$

From the above description it is clear that though there have been moderate data from candidate gene based association testing with SZ among Indian population, neither an Indian GWAS nor a study attempting replication of the Caucasian SZ GWAS findings are available to date. The following report is our first attempt to examine selected SNPs from the previous Caucasian SZ GWAS findings $(p<$ $10^{-8}$ ) or SNPs from other large-scale association studies or cataloged in a SZ gene database ${ }^{42}(p<0.05)$ in a moderate sized SZ cohort from north India. We also tested for the first time their association with cognition in a subset of the same study cohort.

\section{RESULTS}

Of the 2038 samples, 67 individuals with a failure rate of $20 \%$ for SNP assays were removed from the analysis. The demographic details of the remaining 1971 individuals are provided in Table 2. rs245201 from CTXN3-SLC12A2 was not in Hardy-Weinberg equilibrium $(p=0.00004)$ and $r 57597593$ from ZNF804A had $<95 \%$ calls; therefore these SNPs were removed from the analysis. Thus, a total of 1971 individuals and 30 SNPs (26 from chromosome 6 and four from other chromosomes, (Table 3) were taken forward for disease association. 
Table 2. Demographic details of schizophrenia study cohort

(a) Distribution of age and gender in the study cohort

\begin{tabular}{lllll}
\hline $\begin{array}{l}\text { Total } \\
\text { samples }\end{array}$ & $\begin{array}{l}\text { Cases (males/ } \\
\text { females) }\end{array}$ & $\begin{array}{l}\text { Age in } \\
\text { years mean } \\
\pm S D\end{array}$ & $\begin{array}{l}\text { Controls } \\
\text { (males/ } \\
\text { females) }\end{array}$ & $\begin{array}{l}\text { Age in } \\
\text { years mean } \\
\pm S D\end{array}$ \\
\hline 1971 & $980(554 / 426)$ & $30 \pm 8.99$ & $989^{*}(554 / 433)$ & $43 \pm 13.75$
\end{tabular}

(b) Distribution of samples with cognition data

Cases $(n=189) \quad$ Controls $(n=119)$

*No gender information for two individuals

Tests of association

Genotypic. Genotypic association was observed for SNPs rs6916394 T >C $(p=0.02 ; \mathrm{df}=2)$ and rs3130615 T>C $(p=0.06$; $\mathrm{df}=2$ ) both from MICB (Table 3) and none of them withstood multiple testing.

Allelic. Modest allelic association of only one SNP namely rs2064430, AHI1 $(p=0.04)$; and a trend for four SNPs namely

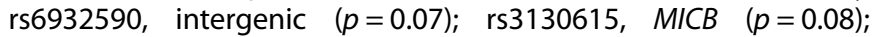
rs6916921, NFKBIL1 $(p=0.08)$ and rs9273012, HLA-DQA1 $(p=$ 0.06 ) was observed with SZ. Detailed results for all the SNPs tested are presented in Table 3 and notably, none of them withstood multiple testing.

Haplotypic. Nominal haplotypic association $(p=0.01-0.05)$ was observed for haplotypes generated in PLINK using 1-6 marker sliding window (Supplementary Table 2) but did not withstand Bonferroni correction. SNPs identified to be associated in the univariate analysis were seen to be the drivers of associated haplotypes.

Association of SNPs with cognition. When the cases were compared with the controls, there was significant association of SZ status with the domains of abstraction $\left(p=1.32 \times 10^{-11}\right)$; attention $(p=0.00002)$; face memory $(p=0.00009)$; spatial memory $\left(p=3.14 \times 10^{-6}\right)$; working memory $\left(p=3.98 \times 10^{-7}\right)$; sensorimotor $(p=0.0002)$ and emotional processing $\left(p=2.04 \times 10^{-6}\right)$ (Supplementary Table 3). Six SNPs were associated with five cognition domains: MICB, rs6916394 with abstraction (0.04); AHI1, rs2064430 and NFKBIL1, rs2230365 ( $p=0.05 ; p=0.04$, respectively) with face memory; rs3130375 with working memory $(p=0.007)$; rs377763 ( $p=0.004)$ with sensorimotor; NFKBIL1, rs6916921 ( $p=$ 0.01 ) with emotion (Table 4). Supplementary Table 1

All genetic and cognitive data used in this study can be accessed at https://figshare.com/s/34a8ffa9182221b075c5.

Modifiers. Epistatic interactions were evaluated for all these SNPs using PLINK. We analyzed several SNPs in close proximity in the chromosome 6p21-24 region. SNP-SNP interactions were observed between rs3828917 (MICB) X rs377763 (Intergenic), rs2071278 $(\mathrm{NOTCH4}) \times$ rs6916394 (Intergenic), rs2071278 (NOTCH4) $\times$ rs2523651 (upstream of MICB), and rs2244839 (HCG26) × rs1800610 (TNF). None of the $p$-values withstood Bonferroni corrections (Table 5).

\section{DISCUSSION}

Prior studies indicate that Indians have a complex population history, with similarities and differences from populations with Caucasian ancestry. Thus, studies in Indian samples might indicate susceptibility loci for SZ that are identical with, or distinct from the prior GWASs that were conducted extensively in Caucasian ancestry samples. The present study is an initial effort to extend the GWAS analyses on Caucasian populations to Indian samples. We focused primarily on the chromosome $6 p$ region, which harbors the strongest and most consistently replicated susceptibility loci in the GWASs. ${ }^{6,16,17,43}$ Our analyses modestly support some of the prior associations, ${ }^{42,}{ }^{43}$ while indicating that there are possibly other associations specific to this population, which remains to be identified.

\section{Allelic/genotypic/hapoltypic associations}

Of the 32 most significant GWAS and other prioritized SNPs, largely from chromosome 6, modest allelic/genotypic association of six SNPs namely rs6932590 (intergenic, located close to another SZ GWAS SNP rs13194053), rs6916921 (NFKBIL1), rs6916394 and rs3130615 (MICB) and rs9273012 (HLA-DQA1) on 6p and rs2064430 (AHI1) on $6 q$ was noted (Table 2). The failure to have strong significance and to replicate other SNPs may reflect insufficient power of the study sample or differences in the genetic architecture of SZ across populations. As for the other five SNPs, though associations were only nominally significant, the findings are notable, because NFKBIL1 and MICB are immune response genes and previously implicated in SZ etiology. ${ }^{16,17,44}$ On the other hand, AHI1 (located on chromosome 6q, has been reported to be associated with SZ in an Israeli Arab population ${ }^{20,45}$; in Palestinian Arab families ${ }^{46}$ and in a meta-analysis of European populations. ${ }^{47}$ More recently, SNPs from this gene have been included in a SZ database (www.szgenes.org). ${ }^{42,}{ }^{43}$ However, this gene has not been found to be associated with SZ in any GWASs or PGC GWAS. In an ab initio analysis, AHI1 was identified as a common helper provirus integration site for murine leukemias and lymphomas. ${ }^{48}$ AHI1 is a component of a protein complex in the basal body present at the base of cilia. This complex serves as a barrier between plasma and ciliary membranes to selectively restrict protein diffusion. Disruption of this complex has been shown to cause various neurological developmental abnormalities, and neurological defects are widely observed in various ciliopathies. ${ }^{49}$ In fact, AHI1 or Jouberin could be important for both cerebellar and cortical development in humans and mutations in this gene have been associated with Joubert syndrome, a brain developmental disorder. Multiple studies indicate that primary cilium is a sensory organelle which gathers extracellular cues that regulate brain development ${ }^{50}$ and may play a critical role in brain patterning. ${ }^{51}$

Further, epistatic interactions tested among all these functionally similar genes such as, MICB, NOTCH4, HLA-DQA1, HCG26 etc. on chromosome 6 , revealed a few modest associations (Table 5). Some nominally significant associations with cognitive domains were noted (Table 3). Initially, impaired cognitive performance was observed among patients with SZ for all cognitive domains except for spatial ability. Further analyses indicated that rs377763 is significantly associated with accuracy in the sensorimotor domain. This SNP is localized between NOTCH4 and BTNL, both of which have been implicated in SZ susceptibility. ${ }^{52,53}$ An interaction of NOTCH4 with cognition and SZ was also reported in another study. ${ }^{54}$ rs3130375, the most significant SNP in an international schizophrenia consortium (ISC) study (comprised of multiple European GWASs) is associated with accuracy in the working memory domain and is localized to RPP21, which is involved in processing of $5^{\prime}$ leader sequences of precursor t-RNA. Variations in this gene are associated with monocyticleukemias.", 24 SNPs in AHI1 and NFKBIL1 are significantly associated with accuracy in the face memory domain. Since these two SNPs were associated with variation in the same cognition domain, i.e., face memory, epistatic interactions were evaluated, revealing a modest association $(p=0.03)$.

It is now known that neurons have primary cilium which may control cerebellar morphogenesis ${ }^{55}$ and defects in these sensory neuronal cilia may cause some neurological diseases, as primary cilia are critical in early brain patterning and formation of adult neural stem cells. ${ }^{50}$ The ciliary protein complex and innate 


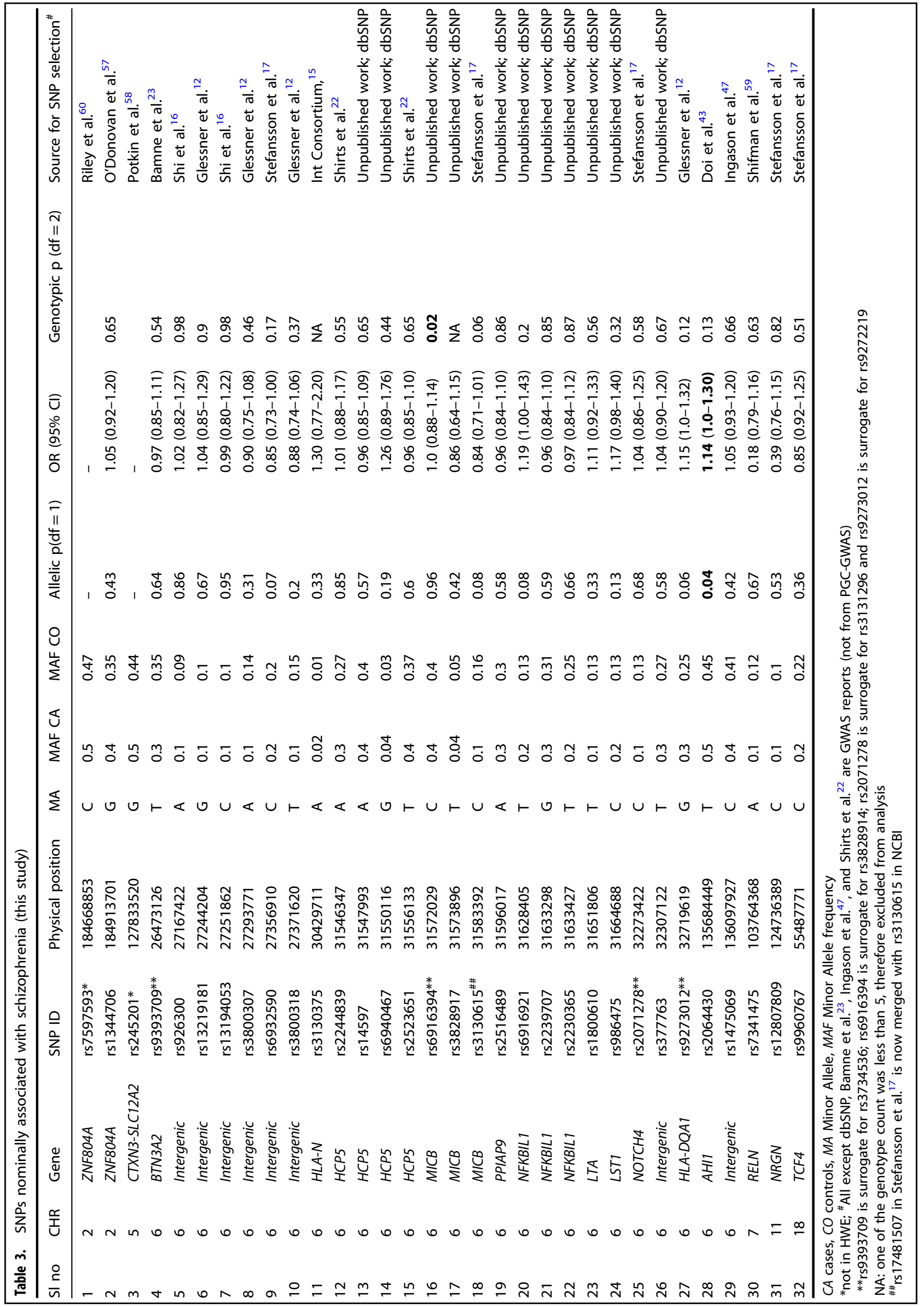


Table 4. Association of chromosome 6 markers with cognition in the study cohort

\begin{tabular}{lllll}
\hline Cognition domain (dependent variable) & Markers (gene/locus) (independent variables) & $B$ value & $p$-value & $95 \% \mathrm{Cl}$ \\
\hline Abstraction & rs6916394 (MICB) & -0.12 & $\mathbf{0 . 0 4}$ & $\mathbf{( - ) 0 . 3 3}$ to (-)0.005 \\
Face memory & rs2064430 (AHI1) & -0.11 & $\mathbf{0 . 0 5}$ & $\mathbf{( - ) 0 . 3 2}$ to (-)0.0002 \\
& rs2230365 (NFKBIL1) & +0.12 & $\mathbf{0 . 0 4}$ & $\mathbf{0 . 0 1 2 - 0 . 4 1}$ \\
Working memory & rs3130375 (Intergenic) & +0.16 & $\mathbf{0 . 0 0 7}$ & $\mathbf{0 . 2 7 - 1 . 7}$ \\
Sensorimotor & rs377763 (Intergenic) & -0.17 & $\mathbf{0 . 0 0 4}$ & $\mathbf{( - ) 0 . 4 7}$ to (-)0.09 \\
Emotion & rs6916921 (NFKBIL1) & +0.15 & $\mathbf{0 . 0 1}$ & $\mathbf{0 . 0 7 - 0 . 5 7}$ \\
\hline
\end{tabular}

Table 5. Shows significant epistatic interactions between chromosome 6 markers

\begin{tabular}{lllll}
\hline Gene (SNP1) & Gene (SNP2) & Odds ratio for interaction & $x^{2}$ (1df) & Asymptotic $p$-value \\
\hline HCG26 (rs2244839) & TNF (rs1800610) & 1.57 & 7.00 & $\mathbf{0 . 0 0 8}$ \\
upstream of MICB (rs2523651) & NOTCH4 (rs2071278) & 1.55 & 7.81 & $\mathbf{0 . 0 0 5}$ \\
MICB (rs6916394) & NOTCH4 (rs2071278) & 1.47 & 6.71 & $\mathbf{0 . 0 1}$ \\
MICB (rs3828917) & Intergenic (rs377763) & 0.37 & 9.44 & $\mathbf{0 . 0 0 2}$ \\
\hline Note: $p$-values $\leq 0.01$ only included & & & & \\
\hline
\end{tabular}

immune response genes might be interacting to keep away the unsolicited entry of pathogens. Variation in the genes governing these functions might render the person susceptible to pathogens, which may in turn disrupt the internal homeostasis and alter synaptic plasticity, thus leading to cognitive deficits. ${ }^{50,55}$ Alternately, since $A H I 1$ is known to have a site for provirus integration and is involved in ciliary machinery that allows differential protein diffusion, and NFKBIL1 is involved in immune response, these genes may be interacting to maintain internal homeostasis by restricting unsolicited pathogen entry. Separately, modest associations were observed with rs6916394 (abstraction domain, $p=0.04, M I C B$ ) and rs6916921 (emotion domain, $p=0.01$, NFKBIL1) but how these SNPs/genes affect these cognitive domains and the role of these genes in cognition is not clear at present. A susceptibility locus conferring vulnerability to SZ with selective impairments on sustained attention on $6 \mathrm{p}$ for haplotype rs1225934-rs13878 in BMP6-TXNDC5 has been reported. ${ }^{56}$

Some limitations of the present analyses are notable. The primary limitation is the absence of genome-wide association data in this or other Indian samples. Based on the present analyses, future GWAS in Indian samples could well identify novel genetic risk factors for SZ, addressing the missing heritability issue. Though it could be argued that some of the analyses represented tests of the prior GWAS results, the novel associations with cognition reported here need to be evaluated further in independently ascertained samples.

In summary, the present study in a north Indian SZ cohort shows very limited replication of predominantly Caucasian population-based associations reported in literature and this may be attributed to the differences in genetic architecture between the two populations or a lack of a large enough sample size. Resequencing the gene dense chromosome 6 region may facilitate identification of the primary risk variants across ethnically different populations and thereby provide new insights into the elusive SZ biology. Our study only weakly supports the prevailing notion that chromosome 6 SNPs and immune genes are involved in cognition decline and people with predilection to viral infections have a higher chance of cognitive decline and are at greater risk for developing SZ, but the probable mechanism underlying this is unclear.

\section{MATERIALS AND METHODS}

\section{Subjects}

The study was performed with Institutional ethical committee approval obtained at the participating centers i.e., Post Graduate Institute of Medical Education and Research-Dr. Ram Manohar Lohia (PGIMER-Dr. RML) Hospital and University of Delhi South Campus, New Delhi. The study was conducted in accordance with all relevant protocols. Initially, Schizophrenia cases $(n=1009)$ and controls $(n=1029)$ were recruited at PGIMER -Dr. RML hospital. Controls included age and gender balanced adult individuals without a history of psychotic illness $(n=507)$ and cord blood samples $(n=482)$. All participants completed the Diagnostic Interview for Genetic Studies (DIGS) and were evaluated according to the DSM IV criteria. ${ }^{38,40}$ Written informed consent was obtained from all participants. Maternal consent was obtained for the cord blood samples. All the subjects were of north Indian origin and are genetically distinct as evidenced by admixture analysis. ${ }^{26}$

\section{Genetic analysis}

Venous blood was obtained from all participants and DNA was extracted using routine phenol chloroform method.

A total of 32 SNPs (Table 3 and Supplementary Table 1) were analyzed. SNPs were selected based on available published and unpublished association studies, also keeping in view the estimated level of polymorphism and available linkage disequilibrium (LD) structure among Indians. Of these, 26 SNPs were from chromosome 6 (Supplementary Table 1). The remaining SNPs (ZNF804A: rs1344706 and rs7597593, CTXN3SLC12A2: rs245201, RELN: rs7341475, NRGN: rs12807809, TCF4: rs9960767 were localized to chromosomes $2,5,7,11$, and 18 , respectively. Of the 26 SNPs (Supplementary Fig. 1), 10 SNPs $\left(p \leq 10^{-8}\right)$ were drawn from the Caucasian SZ GWAS ${ }^{13}, 16,17,57-60$ and 16 SNPs were from other published and unpublished association studies in Caucasian and African-American populations. ${ }^{23}$ Of note, chromosome $6 p$ has been the most consistently associated region not only in the Caucasian SZ GWASs but also with cognition $^{61}$ and immune dysfunction. ${ }^{62}$ Since our study focused on $S Z$ and cognition, these SNPs were selected. All these SNPs had minor allele frequencies (MAF) $\geq 0.01$ in the Indian samples. SNP assays were performed using Sequenom mass array according to manufacturer's protocol, at a commercial facility. Genotypes were called using MassARRAY TYPER 4.0 genotyping software. All questionable calls were rechecked and called manually. Multidimensional scaling using PLINK indicated that the cases and controls were similar with regard to population structure (Supplementary Fig. 2). 
Cognitive data

Cognitive functions were assessed using the Hindi version of the Penn Computerized Neuropsychological Battery $\left(\mathrm{CNB}^{63}{ }^{63}\right)$ as described elsewhere ${ }^{64}$ Accuracy index of eight domains of cognition namely abstraction and mental flexibility, attention, facial memory, spatial memory, working memory, spatial ability, sensorimotor, and emotional equity were assessed in this study.

\section{Statistical analysis}

Hardy-Weinberg equilibrium (HWE) checks and LD analysis was conducted using Haploview software ${ }^{65}$ (http://www.broadinstitute.org/scientific-community/science/programs/medical-and-populationgenetics/haploview/haploview) and PLINK(http://pngu.mgh.harvard.edu/ purcell/plink/). Allelic, genotypic, and haplotypic associations were analyzed using PLINK. Allele and genotype frequencies of each SNP were compared between patients and control groups. The minor allele was taken as a reference for allelic association (df 1); and the three genotypes formed by the two alleles were considered for genotypic associations (df 2). Pearson's chi-square tests and odds ratios (OR) estimations at $95 \%$ confidence intervals (Cl) were performed. For haplotypic association, a sliding window of 2 to 6 SNP combinations based on their consecutive physical location along a chromosome or chromosomal segment was used with default parameters. Power of the SNPs in the study was calculated using log additive inheritance model in QUANTO software (http://hydra.usc.edu/gxe/). The sample has $80 \%$ power to detect associations with SZ risk having an OR $\geq 1.4$, for SNPs having minor allele frequencies (MAF) $>20 \%$, with a disease prevalence of $1 \%$.

Cases and controls were initially compared for demographic variables and cognitive functions using univariate analyses. Subsequently, linear regression analyses for individual cognitive domains were conducted to assess association between SNPs, cognitive domains, and health status using SPSS version 21. Since age has a significant association with cognitive function, the cognitive domains were adjusted for age.

\section{Data availability}

All genetic and cognitive data used in this study can be accessed at https:// figshare.com/s/34a8ffa9182221b075c5.

\section{ACKNOWLEDGEMENTS}

We are grateful for the sample collection by trained and dedicated staff at Dr. RML hospital, New Delhi; DNA isolation by Mrs. Anjali Dabral and valuable imputs from Mr. Jibin John and Ms. Upasana Bhattacharya at the Department of Genetics, University of Delhi, South Campus (UDSC), New Delhi and M/s Aceprobe Technologies, New Delhi, for facilitating the genotyping work. We acknowledge infrastructure support provided by the University Grants Commission (UGC), New Delhi, through Special Assistance Programme and Department of Science and Technology, New Delhi, through FIST and DU-DST PURSE programmes to the Department of Genetics, University of Delhi, South Campus. This work was supported in part by grants from the Department of Biotechnology, Govt. of India, New Delhi to B.K.T., S.N.D. (\#BT/MB/Project/Schizophrenia/2012-2013); \#BT/PR2425/Med13/089/2001); JC Bose fellowship (\#SR/S2/JCB44/2011) to B.K.T. from the Department of Science and Technology, Government of India; University Grants Commission, New Delhi, one-time grant (\#F19-140/2014(BSR)) to B.K.T. and for DSK-PDF to P.K. (\#BL/13-14/0404); National Institute of Mental Health and Stanley Medical Research Institute, (07R-1712), National Institute of Mental Health (\#MH63480) USA to V.L.N. The funding agencies are not responsible for the design and conduct of the study; collection, management, analysis, or interpretation of the data; nor for preparation, review, or approval of the manuscript.

\section{AUTHOR CONTRIBUTIONS}

B.K.T., S.N.D., and V.L.N. designed the study and wrote the protocol. S.P. prioritized and genotyped the SNPs in the study, performed statistical analysis and wrote the first draft of the manuscript. S.N.D. and her team provided the research samples and T.B. did the cognition assessment and statistical analysis; P.K. contributed to the statistical analysis and preparation of the manuscript. All authors contributed to and have approved the final manuscript.

\section{ADDITIONAL INFORMATION}

Supplementary Information accompanies the paper on the npj Schizophrenia website (doi:10.1038/s41537-017-0030-8).
Competing interests: The authors declare that they have no competing financial interests.

Publisher's note: Springer Nature remains neutral with regard to jurisdictional claims in published maps and institutional affiliations.

\section{REFERENCES}

1. Gottesman, I. I., Laursen, T. M., Bertelsen, A. \& Mortensen, P. B. Severe mental disorders in offspring with 2 psychiatrically ill parents. Arch. Gen. Psychiatry 67, 252 (2010).

2. Lichtenstein, P. et al. Common genetic determinants of schizophrenia and bipolar disorder in Swedish families: a population-based study. Lancet 373, 234-239 (2009).

3. Lewis, C. M. et al. Genome scan meta-analysis of schizophrenia and bipolar disorder, part II: schizophrenia. Am. J. Hum. Genet. 73, 34-48 (2003).

4. Levinson, D. F., Levinson, M. D., Segurado, R. \& Lewis, C. M. Genome scan metaanalysis of schizophrenia and bipolar disorder, part I: methods and power analysis. Am. J. Hum. Genet. 73, 17-33 (2003).

5. Jia, P. et al. Network-assisted investigation of combined causal signals from genome-wide association studies in schizophrenia. PLoS Comput. Biol. 8, e1002587 (2012).

6. Ripke, S. et al. Genome-wide association analysis identifies 13 new risk loci for schizophrenia. Nat. Genet. 45, 1150-1159 (2013).

7. Purcell, S. M. et al. Common polygenic variation contributes to risk of schizophrenia and bipolar disorder. Nature 460, 748 (2009).

8. $\mathrm{Ng}, \mathrm{M}$. Y. M. et al. Meta-analysis of 32 genome-wide linkage studies of schizophrenia. Mol. Psychiatry 14, 774-785 (2009).

9. Bray, N. J. Gene expression in the etiology of schizophrenia. Schizophr. Bull. 34 , 412-418 (2007).

10. Chu, T. T. \& Liu, Y. An integrated genomic analysis of gene-function correlation on schizophrenia susceptibility genes. J. Hum. Genet. 55, 285-292 (2010).

11. Sinkus, M. L., Adams, C. E., Logel, J., Freedman, R. \& Leonard, S. Expression of immune genes on chromosome 6p21.3-22.1 in schizophrenia. Brain Behav. Immun. 32, 51-62 (2013).

12. Glessner, J. T. \& Hakonarson, H. Common variants in polygenic schizophrenia. Genome Biol. 10, 236 (2009).

13. Ripke, S. et al. Biological insights from 108 schizophrenia-associated genetic loci. Nature 511, 421-427 (2014).

14. Giegling, l. et al. Genetics of schizophrenia: a consensus paper of the WFSBP Task Force on Genetics. World J. Biol. Psychiatry 1-14, doi:10.1080/15622975.2016. 1268715 (2017).

15. International Schizophrenia Consortium, I. S. et al. Common polygenic variation contributes to risk of schizophrenia and bipolar disorder. Nature 460, 748-752 (2009).

16. Shi, J. et al. Common variants on chromosome $6 \mathrm{p} 22.1$ are associated with schizophrenia. Nature 460, 753 (2009).

17. Stefansson, $\mathrm{H}$. et al. Common variants conferring risk of schizophrenia. Nature 460, 744-747 (2009).

18. Sekar, A. et al. Schizophrenia risk from complex variation of complement component 4. Nature 530, 177-183 (2016).

19. Cross-Disorder Group of the Psychiatric Genomics Consortium. Identification of risk loci with shared effects on five major psychiatric disorders: a genome-wide analysis. Lancet 381, 1371-1379 (2013).

20. Amann-Zalcenstein, D. et al. AHI1, a pivotal neurodevelopmental gene, and C6orf217 are associated with susceptibility to schizophrenia. Eur. J. Hum. Genet. 14, 1111-1119 (2006).

21. Prior, M. J. et al. The characterization of Abelson helper integration site-1 in skeletal muscle and its links to the metabolic syndrome. Metabolism 59, 1057-1064 (2010).

22. Shirts, B. H. et al. Polymorphisms in MICB are associated with human herpes virus seropositivity and schizophrenia risk. Schizophr. Res. 94, 342-353 (2007).

23. Bamne, M. et al. Evaluation of HLA polymorphisms in relation to schizophrenia risk and infectious exposure. Schizophr. Bull. 38, 1149-1154 (2012).

24. Mokhtari, R. \& Lachman, H. M. The major histocompatibility complex (MHC) in schizophrenia: a review. J. Clin. Cell Immunol. 7, 479 (2016).

25. Gur, R. E. Neuropsychiatric aspects of schizophrenia. CNS Neurosci. Ther. 17, 45-51 (2011).

26. Blokland, G. A. M. et al. Heritability of neuropsychological measures in schizophrenia and nonpsychiatric populations: a systematic review and meta-analysis. Schizophr. Bull. doi:10.1093/schbul/sbw146 (2016).

27. Gejman, P. V., Sanders, A. R. \& Duan, J. The role of genetics in the etiology of schizophrenia. Psychiatr. Clin. North Am. 33, 35-66 (2010). 
28. Basu, A., Sarkar-Roy, N. \& Majumder, P. P. Genomic reconstruction of the history of extant populations of India reveals five distinct ancestral components and a complex structure. Proc. Natl Acad. Sci. 113, 1594-1599 (2016).

29. Reich, D., Thangaraj, K., Patterson, N., Price, A. L. \& Singh, L. Reconstructing Indian population history. Nature 461, 489-494 (2009).

30. Thangaraj, K. et al. In situ origin of deep rooting lineages of mitochondrial Macrohaplogroup ' $M$ ' in India. BMC Genom. 7, 151 (2006).

31. Juyal, G. et al. Population and genomic lessons from genetic analysis of two Indian populations. Hum. Genet. 133, 1273-1287 (2014).

32. Negi, S. et al. A genome-wide association study reveals ARL15, a novel non-HLA susceptibility gene for rheumatoid arthritis in North Indians. Arthr. Rheum. 65, 3026-3035 (2013).

33. Ganguli, H. C. Epidemiological findings on prevalence of mental disorders in India. Indian J. Psychiatry 42, 14 (2000).

34. Kukshal, P., Thelma, B. K., Nimgaonkar, V. L. \& Deshpande, S. N. Genetics of schizophrenia from a clinicial perspective. Int. Rev. Psychiatry 24, 393-404 (2012).

35. Thelma, B., Srivastava, V. \& Tiwari, A. K. Genetic underpinnings of tardive dyskinesia: passing the baton to pharmacogenetics. Pharmacogenomics 9, 1285-1306 (2008).

36. Verma, R., Kubendran, S., Das, S. K., Jain, S. \& Brahmachari, S. K. SYNGR1 is associated with schizophrenia and bipolar disorder in southern India. J. Hum. Genet. 50, 635-640 (2005).

37. Jajodia, A. et al. Evidence for schizophrenia susceptibility alleles in the Indian population: an association of neurodevelopmental genes in case-control and familial samples. Schizophr. Res. 162, 112-117 (2015).

38. Kukshal, P. et al. Association study of Neuregulin-1 gene polymorphisms in a north Indian schizophrenia sample. Schizophr. Res. 144, 24-30 (2013).

39. Ohi, K., Shimada, T., Yasuyama, T., Uehara, T. \& Kawasaki, Y. Variability of 128 schizophrenia-associated gene variants across distinct ethnic populations. Transl. Psychiatry 7, e988 (2017)

40. John, J. et al. Association study of MiRSNPs with schizophrenia, tardive dyskinesia and cognition. Schizophr. Res. 174, 29-34 (2016).

41. John, J. et al. Possible role of rare variants in Trace amine associated receptor 1 in schizophrenia. Schizophr. Res. doi:10.1016/j.schres.2017.02.020 (2017).

42. Allen, N. C. et al. Systematic meta-analyses and field synopsis of genetic association studies in schizophrenia: the Sz Gene database. Nat. Genet. 40, 827-834 (2008).

43. Doi, N. et al. Paradox of schizophrenia genetics: is a paradigm shift occurring? Behav. Brain Funct. 8, 28 (2012).

44. Pouget, J. G. et al. Genome-wide association studies suggest limited immune gene enrichment in schizophrenia compared to 5 autoimmune diseases. Schizophr. Bull. 42, 1176-1184 (2016).

45. Ingason, A. et al. Support for involvement of the AHI1 locus in schizophrenia. Eur. J. Hum. Genet. 15, 988-991 (2007).

46. Torri, F. et al. Fine mapping of $\mathrm{AHI} 1$ as a schizophrenia susceptibility gene: from association to evolutionary evidence. FASEB J. 24, 3066-3082 (2010).

47. Ingason, A. et al. A large replication study and meta-analysis in European samples provides further support for association of AHI markers with schizophrenia. Hum. Mol. Genet. 19, 1379-1386 (2010).

48. Poirier, Y. \& Jolicoeur, P. Distinct helper virus requirements for Abelson murine leukemia virus-induced pre-B- and T-cell lymphomas. J. Virol. 63, 2088-2098 (1989).

49. Valente, E. M., Rosti, R. O., Gibbs, E. \& Gleeson, J. G. Primary cilia in neurodevelopmental disorders. Nat. Rev. Neurol. 10, 27-36 (2013).

50. Han, Y.-G. \& Alvarez-Buylla, A. in Two Faces of Evil: Cancer and Neurodegeneration, [Research and Perspectives in Alzheimer's Disease book series (ALZHEIMER)]. Chapter: Primary Cilia as Switches in Brain Development and Cancer, 73-82 (Springer Berlin Heidelberg). doi:10.1007/978-3-642-16602-0_6 (2011).

51. Gorivodsky, M. et al. Intraflagellar transport protein 172 is essential for primary cilia formation and plays a vital role in patterning the mammalian brain. Dev. Biol. 325, 24-32 (2009).

52. Wang, Z. et al. A review and re-evaluation of an association between the NOTCH4 locus and schizophrenia. Am. J. Med. Genet. B Neuropsychiatr. Genet. 141B, 902-906 (2006).

53. Abeler-D örner, L., Swamy, M., Williams, G., Hayday, A. C. \& Bas, A. Butyrophilins: an emerging family of immune regulators. Trends Immunol. 33, 34-41 (2012).

54. Wassink, T. H., Nopoulos, P., Pietila, J., Crowe, R. R. \& Andreasen, N. C. NOTCH4 and the frontal lobe in schizophrenia. Am. J. Med. Genet. B Neuropsychiatr. Genet 118B, 1-7 (2003).
55. Chizhikov, V. V. et al. Cilia proteins control cerebellar morphogenesis by promoting expansion of the granule progenitor pool. J. Neurosci. 27, 9780-9789 (2007).

56. Lin, S.-H. et al. Clustering by neurocognition for fine mapping of the schizophrenia susceptibility loci on chromosome 6p. Genes Brain Behav. 8, 785-794 (2009).

57. O'Donovan, M. C., Craddock, N. J. \& Owen, M. J. Genetics of psychosis; insights from views across the genome. Hum. Genet. 126, 3-12 (2009).

58. Potkin, S. G. et al. A genome-wide association study of schizophrenia using brain activation as a quantitative phenotype. Schizophr. Bull. 35, 96-108 (2009).

59. Shifman, S. et al. Genome-wide association identifies a common variant in the reelin gene that increases the risk of schizophrenia only in women. PLoS Genet. 4, e28 (2008).

60. Riley, B. et al. Replication of association between schizophrenia and ZNF804A in the irish case-control study of schizophrenia sample. Mol. Psychiatry 15, 29-37 (2010).

61. Walters, J. T. R. et al. The role of the major histocompatibility complex region in cognition and brain structure: a schizophrenia GWAS follow-up. Am. J. Psychiatry 170, 877-885 (2013).

62. Avramopoulos, D. et al. Infection and inflammation in schizophrenia and bipolar disorder: a genome wide study for interactions with genetic variation. PLOS ONE 10, e0116696 (2015)

63. Gur, R. et al. Computerized neurocognitive scanning: I. methodology and validation in healthy people. Neuropsychopharmacology 25, 766-776 (2001).

64. Bhatia, T. et al. Adjunctive cognitive remediation for schizophrenia using yoga: an open, non-randomised trial. Acta Neuropsychiatr. 24, 91-100 (2012).

65. Barrett, J. C., Fry, B., Maller, J. \& Daly, M. J. Haploview: analysis and visualization of LD and haplotype maps. Bioinformatics 21, 263-265 (2005).

66. Srinivas, L. et al. Pro-inflammatory cytokines and their epistatic interactions in genetic susceptibility to schizophrenia. J. Neuroinflammation 13, 105 (2016).

67. Sujitha, S. P. et al. DNA repair gene (XRCC1) polymorphism (Arg399GIn) associated with schizophrenia in South Indian population: a genotypic and molecular dynamics study. PLOS ONE 11, e0147348 (2016).

68. Saradalekshmi, K. R. et al. DNA methyl transferase (DNMT) gene polymorphisms could be a primary event in epigenetic susceptibility to schizophrenia. PLOS ONE 9, e98182 (2014).

69. Kukshal, P. et al. Dopaminergic gene polymorphisms and cognitive function in a north Indian schizophrenia cohort. J. Psychiatr. Res. 47, 1615-1622 (2013).

70. Srivastava, V., Deshpande, S. N. \& Thelma, B. K. Dopaminergic pathway gene polymorphisms and genetic susceptibility to schizophrenia among north Indians. Neuropsychobiology 61, 64-70 (2010).

71. Gupta, M. et al. Genetic susceptibility to schizophrenia: role of dopaminergic pathway gene polymorphisms. Pharmacogenomics 10, 277-291 (2009).

72. Vijayan, N. N. et al. Evidence of association of serotonin transporter gene polymorphisms with schizophrenia in a South Indian population. J. Hum. Genet. 54, 538-542 (2009)

73. Talkowski, M. E. et al. Novel, replicated associations between dopamine D3 receptor gene polymorphisms and schizophrenia in two independent samples. Biol. Psychiatry 60, 570-577 (2006).

74. Prasad, S. et al. Association analysis of NOTCH 4 polymorphisms with schizophrenia among two independent family based samples. Am. J. Med. Genet. B Neuropsychiatr. Genet. 131B, 6-9 (2004).

75. Saleem, Q. et al. Association of CAG repeat loci on chromosome 22 with schizophrenia and bipolar disorder. Mol. Psychiatry 6, 694-700 (2001).

Open Access This article is licensed under a Creative Commons Attribution 4.0 International License, which permits use, sharing, adaptation, distribution and reproduction in any medium or format, as long as you give appropriate credit to the original author(s) and the source, provide a link to the Creative Commons license, and indicate if changes were made. The images or other third party material in this article are included in the article's Creative Commons license, unless indicated otherwise in a credit line to the material. If material is not included in the article's Creative Commons license and your intended use is not permitted by statutory regulation or exceeds the permitted use, you will need to obtain permission directly from the copyright holder. To view a copy of this license, visit http://creativecommons. org/licenses/by/4.0/.

(c) The Author(s) 2017 